\title{
Editorial
}

\section{Digital Health During COVID-19 Pandemic and Beyond}

\author{
Rahman $\mathrm{SMM}^{1 *}$, Hossain $\mathrm{SM}^{2}$, Jahan $\mathrm{MU}^{3}$ \\ ${ }^{1}$ Institute of Public Health, Dhaka, Bangladesh; ${ }^{2}$ Directorate General of Health Services, Dhaka, Bangladesh; \\ ${ }^{3}$ Bangladesh Medical Research Council, Dhaka, Bangladesh
}

Coronavirus disease (COVID-19) was unknown before the outbreak began in Wuhan, China in December 2019. The virus rapidly spread in different countries across the globe. The World Health Organization (WHO) declared it as a pandemic on the $11^{\text {th }}$ March $2020 .{ }^{1}$ The pandemic has revealed many areas of public health preparedness those are lacking both in developed and developing countries. Digital interventions provide many opportunities for strengthening health systems. ${ }^{2}$ It could be a vital resources and could play a critical role in global response to this public health emergency.

Digital health is the use of digital information and communication technologies for efficient and timely delivery of health care services, aimed at promoting and protecting the health of the individuals and communities. Furthermore, digital technologies are also being used for conducting monitoring and surveillance of the health programmes, health education, research, development of human resources including continued professional development, risk analysis speacially the risk commucation. Data being generated through services, research, monitoring and surveillance are also used for health management and decision making.

Technologies are used in digital health include both hardware and software including telemedicine, telehealth, mobile health ( $\mathrm{m}$ healht), e-mail, text message, social media, warable devices, web based analysis, clinic or remote monitoring sensors, health information technogy and others. ${ }^{3,4}$ Digital health is not only being implemented by the health professionals, it is a multi-disciplinary domain involving a number of stakeholders, including clinicians, researchers and scientists with a wide range of expertise in healthcare, engineering, social sciences, public health, health economics and data management. ${ }^{5}$

Bangladesh has been implementing the digital health services to promote and protect public health, including telehealth, video-consultation, reporting even through data collection from the remote rural areas, monitoring, survilance, human resource devlopement including continued professional

*Correspondence: Dr. Shah Md. Mahfuzur Rahman, Institute of Public Health Mohakhali, Dhaka-1212, Bangladesh; e-mail: smahfuzbd@gmail.com; ORCID: 00000002-5069-6718 development etc since long before the COVID-19 pandemic. During the COVID-19 pandemic, additional services have been added including risk communications, contact tracing and hot spot indentifaication etc, and stregnthening of the telemedicine and other pre-existing services. In addition to the public sector, the private sectors also joined hands on the digital health services partcicularly tele-medicine, viedo-consultation and others, to serve the population at need.

The government of Bangladesh is well committed to providing equal access to quality health care to all. Digital health is a critical enabler for the overall transformation of the health system. Hence, mainstreaming telemedicine in health system will minimise inequality and barriers to access health care. In July 2020, the Bangladesh has developed telemedicine guidelines, which will be a key enabler in fostering the growth of telemedicine which is being utilised by more healthcare practitioners and patients in a wide variety of forms. ${ }^{6}$ The World Health Organization spelled out in its global strategy for digital health, that the use of digital technologies should be safe, ethical, cost-effective and affordable; and it should be people-centred, evidence-based, effective, efficient, sustainable, inclusive, equitable and contextualised. ${ }^{7}$

Thus, it is suggested that the health authorities should expand and strengthen the digital health, for making it an integral part of the health systems, during and beyond the COVID-19 pandemic. Based on the experience prior to and during the pandemic, digital health is to be used to promote and protect public health through providing promotive, preventive, curative, and rehabilitative services, as well as real time data sharing, monitoring and surveillance, human resource development including continued professional development, and timely and effective response to the public health emergencies. Its use may be further extended to effective and timely communication with the media, public, decision makers, professional bodies and others. All these efforts will facilitate the progress in attaining the sustainable development goals. 


\section{References}

1. Rahman SMM, Hossain SM, Jahan MU. COVID-19 in Bangladesh: Measures for containment. Editorial. Bangladesh Medical Res Counc Bull 2020;46:01-02.

Doi:10.3329/bmrc.v46i1.47460

2. Mahmood S, Hasan K, Caras MC, Labrique A. Global preparedness against COVID-19: We must leverage the powers of digital health. JMIR Public Health and Surveillance. 2020;6:e18980. Doi:10.2196/18980

3. Ronquillo Y, Meyers A; Korvek SJ. Digital Health. CBI Bookshelf. A service of the National Library of Medicine, National Institutes of Health. StatPearls [Internet]. Treasure Island (FL): StatPearls Publishing; January 2020.
4. Digital Health Center of Excellence. US Food \& Drug Administration. https://www.fda.gov/ medical-devices/digital-health-center-excellence

5. O’Donoghue J, Herbert J. Data Management within mHealth Environments: Patient Sensors, Mobile Devices, and Databases. Journal of Data and Information Quality. 2012;4: 1-20. Doi:10.1145/2378016.2378021. S2CID 2318649.

6. Bangladesh Medical \& Dental Council (BMDC). Telemedicine Guidelines. BMDC, Dhaka, July 2020.

7. World Health Organization. Draft global strategy on digital health 2020-2025. WHO, Geneva. July 2020. 